\title{
SISTEMAS DE CONTROLE GERENCIAL E O PROCESSO DE INOVAÇÃO
}

\section{Edelcio Koitiro Nisiyama}

Mestre em Controladoria Empresarial pelo Programa de Pós-Graduação em Ciências Contábeis da Universidade Presbiteriana Mackenzie - MACKENZIE

Professor do Insper - Instituto de Ensino e Pesquisa

E-mail: edelcio.kn@ terra.com.br (Brasil)

\section{José Carlos Tiomatsu Oyadomari}

Professor do Programa de Mestrado Profissional em Ciências Contábeis da Universidade Presbiteriana Mackenzie - MACKENZIE

Doutorado em Controladoria e Contabilidade pela Universidade de São Paulo - USP

E-mail: oyadomari@mackenzie.br (Brasil)

\section{RESUMO}

Esta pesquisa tem como objetivo identificar as contribuições dos estudos empíricos internacionais para o desenvolvimento de pesquisas brasileiras na análise dos relacionamentos entre o Sistema de Controle Gerencial (SCG) e a inovação. A inovação nas empresas é um processo e como tal precisa ser gerenciado. Essa necessidade de gestão da inovação enfatiza a relevância dos sistemas de controle, pois a adequação dos controles gerenciais nos diferentes contextos estratégicos pode ser fundamental para o sucesso das organizações. Este artigo faz uma breve revisão do modelo Levers of Control de Simons (1995) que é um dos modelos de gestão organizacional mais abrangentes. Os sistemas definidos por Simons (1995) possibilitam melhor entendimento da aplicação dos controles gerenciais. Ressaltando a importância da inovação na gestão organizacional, o artigo apresenta uma breve definição de inovação e faz uma revisão da literatura recente publicada nos principais periódicos internacionais envolvendo sistemas de controles gerenciais e o processo de inovação. Saliente-se a ausência do tema na literatura nacional, e internacionalmente percebe-se um número crescente de pesquisas, entretanto os resultados ainda são difusos, proporcionando a possibilidade de explorar novas oportunidades de pesquisas. Nesse contexto, o framework lançado por Davila, Foster \& Oyon (2009b) para análises dos controles e inovações sob a perspectiva estratégica propicia uma estrutura para avançar nas pesquisas visando a formas de controle gerencial que possam estimular o processo de inovação e consequentemente gerar efeito positivo no desempenho organizacional.

Palavras-chave: Inovação; Sistema de Controle Gerencial; Levers of Control. 


\section{INTRODUÇÃO}

Controles são obstáculos para inovação? A empresa pode reduzir ou eliminar os controles? Há controles que ajudam nas inovações? Essas são questões que costumam inquietar os executivos no mundo globalizado e cada vez mais competitivo. A sustentação da vantagem competitiva exige que as organizações inovem continuamente para criar novos produtos, serviços e processos (Kaplan \& Norton, 2004). A inovação como condição para a sustentabilidade das organizações tem sido cada vez mais discutida e entendida como fundamental para competitividade das empresas. Tradicionalmente os Sistemas de Controle Gerencial (SCG) têm sido associados com organizações mecanicistas e frequentemente percebidos como um obstáculo para qualquer inovação e esforço de mudança na organização (Davila, 2005). A teoria recente e estudos empíricos têm questionado essas premissas comumente adotadas sobre o efeito negativo dos SCG na inovação. Nesse novo enfoque, a inovação não é um evento exógeno randômico que ocorre em certas organizações, mas a inovação é vista como um processo organizacional suscetível à gestão, o que explica por que algumas organizações são mais bem-sucedidas do que outras (Davila, 2005).

O entendimento dos SCG tem evoluído muito ao longo dos últimos vinte anos, partindo de uma visão de um assunto meramente técnico e padronizado para uma visão estratégica como instrumento fundamental para gestão dos negócios. Hall (2010), em um ensaio teórico no qual discute a informação contábil e a função gerencial, afirma que o que determina a relevância da informação contábil não é o seu desenho em si, mas a forma como os gestores utilizam e interpretam a informação contábil, por meio de comunicações verbais frutos da interação com os gestores. Portanto, a relevância da informação contábil é determinada pelos gestores no uso rotineiro, mostrando que os usuários não são meramente leitores passivos, mas que há uma forte interação dos usuários com os desenvolvedores das informações (Hall, 2010). Nesse contexto, é relevante buscar aprofundar o conhecimento dos impactos dos controles gerenciais nas inovações, criando um novo paradigma em relação à utilização dos controles gerenciais.

Neste texto, entende-se que controle gerencial é o processo pelo qual os gestores influenciam os outros membros de uma organização para implementar as estratégias da organização (Anthony \& Govidarajan, 2008). As estratégias definem como as organizações devem usar seus recursos para atingir seus objetivos (Merchant \& Van Der Stede, 2007). O controle gerencial inclui todos os dispositivos e sistemas que os gerentes usam para assegurar que os comportamentos e decisões de seus funcionários estão consistentes com os objetivos e estratégias da organização. Esses sistemas são comumente conhecidos como Sistemas de Controle Gerencial (Merchant \& Van Der Stede, 2007).

Revista de Administração e Inovação, São Paulo, v. 9, n. 1, p.106-125, jan./mar. 2012. 
Ressalte-se o papel dos SCG na execução das estratégias da organização. Apesar de existirem diversas definições, existe uma concordância genérica de que a estratégia descreve a direção geral na qual a organização planeja se mover para obter suas metas (Anthony \& Govidarajan, 2008). Tanto a estratégia corporativa como a estratégia das unidades de negócios da organização, comumente conhecida como estratégia competitiva, direcionam o foco da organização e constituem um importante aspecto a ser considerado no projeto de um SCG. A estratégia competitiva da organização define como ela escolhe competir em seu mercado e tenta obter uma vantagem competitiva em relação aos seus competidores. A base fundamental do desempenho acima da média a longo prazo é a vantagem competitiva sustentável (Porter, 1989). Qualquer que seja a estratégia adotada pela organização, a sua sobrevivência no mundo competitivo depende de criar e manter vantagens competitivas. Nesse ambiente, a inovação tornou-se uma fonte de vantagem competitiva a que os protagonistas se referem a fim de competir na arena global (Davila \& Oyon, 2009).

Este artigo tem como objetivo evidenciar as principais contribuições dos estudos empíricos internacionais para o desenvolvimento de pesquisas brasileiras para analisar os relacionamentos entre os controles gerenciais e a inovação. Apesar dos temas inovação e controles estarem presentes em muitos estudos, a literatura nacional carece de pesquisas que analisem as relações entre os controles gerenciais e o processo de gestão da inovação. Foram analisadas diversas pesquisas empíricas publicadas em relevantes periódicos internacionais que podem fornecer importantes evidências para futuras pesquisas. Notadamente, mostra-se o framework desenvolvido por Davila et al. (2009b) para examinar os SCG para os diferentes tipos de contextos de inovação. A estrutura conceitual de Davila define que os esforços de inovação incremental e radical exigem diferentes sistemas de controle gerencial, e que diferentes sistemas são necessários para administrar a criatividade vinda da alta administração e a criatividade que surge do resto da organização (Davila et al., 2009b).

O restante deste artigo está organizado em cinco seções. O modelo teórico Levers of Control de Simons (1995) é apresentado na seção 2, na sequência, os conceitos de inovação são expostos na seção 3 e o framework de Davila et al. (2009b), na seção 4. Na seção 5, é feita uma revisão dos principais estudos recentes sobre o tema e na seção 6 é apresentada uma síntese dos estudos analisados. As considerações finais estão na seção 7 .

\subsection{O MODELO DE SIMONS (LEVERS OF CONTROL)}

Modelo de gestão é a forma como os administradores decidem gerir seus negócios. O modelo de gestão e seu correspondente arcabouço de controle devem proporcionar condições de 
disponibilização de informações que permitirão o desenvolvimento do processo de gestão (Frezatti, Rocha, Nascimento, \& Junqueira, 2009). Um dos modelos mais abrangentes é o modelo de Simons com sua proposta de alavancas de controle. Simons (1995) define seu modelo com base em quatro sistemas:

- Sistema de Crenças - para inspirar e direcionar as procuras por novas oportunidades

- Sistema de Restrições - para estabelecer limites no comportamento nessas procuras

- Sistema de Controle Diagnóstico - para motivar, monitorar e recompensar pelo cumprimento de metas

- Sistema de Controle Interativo - para estimular o aprendizado organizacional e o surgimento de novas ideias e estratégias.

Os sistemas de crenças e de controle interativo criam um ambiente informacional positivo que encoraja o compartilhamento de informações e aprendizado, enquanto os sistemas de restrições e de controle diagnóstico são usados para restringir o comportamento em busca de oportunidades e para a alocação de recursos escassos (Simons, 2000). Simons (1995) explica que o sistema formal de crenças é um conjunto explícito de definições organizacionais que pode ser divulgado em documentos, tais como as declarações de missão da empresa, contendo os valores básicos, propósito e direção para a organização. O sistema de restrições, por sua vez, estabelece limites na procura de oportunidades com base nos riscos de negócios definidos pela empresa. O sistema de restrições mais básico nas organizações é aquele caracterizado pelo código de conduta que normalmente estabelece aspectos comportamentais normativos. Os sistemas de crenças e de restrições são a base para a gestão de riscos estratégicos da organização. As outras duas alavancas de controle são os sistemas de controle diagnóstico e interativo. Simons (2000) sintetiza que os sistemas de controle diagnóstico são usados para comunicar as variáveis críticas de desempenho e para monitorar a implementação das estratégias planejadas, enquanto os sistemas de controle interativo são usados para focar a atenção da organização nas incertezas estratégicas e para ajustar e alterar a estratégia de acordo com as alterações do mercado competitivo. Diferentemente dos sistemas diagnósticos, os sistemas de controle interativos fornecem aos gerentes ferramentas para influenciar a experimentação e busca de oportunidades que possam resultar em estratégias emergentes (Simons, 1995). Os controles interativos alertam a administração para as incertezas de natureza estratégica - problemas ou oportunidades. Isso se torna a base para os gestores adaptarem-se mais rapidamente a mudanças ambientais pensando em novas estratégias (Anthony \& Govidarajan, 2008). Segundo Otley (2003), os controles interativos existem para questionar continuamente se as estratégias existentes ainda são apropriadas. $\mathrm{O}$ uso interativo dos 
sistemas de controle essencialmente desempenha o papel de advogado do diabo para assegurar que as estratégias sejam robustas (Otley, 2003). De forma global, o modelo de Simons enfatiza a relevância do uso interativo do SCG para fomentar a inovação bem-sucedida (Bisbe \& Otley, 2004). A Figura 1 mostra que cada uma das alavancas de controle tem um propósito diferente no controle da estratégia.

Oyadomari, Frezatti, Cardoso, e Aguiar (2009) analisaram os trabalhos que utilizaram o modelo de Simons através de busca em três dos principais periódicos de Contabilidade Gerencial (Accounting, Organizations and Society, Journal of Management Accounting Research e Management Accounting Research) e no SSRN no período de 1995 a 2007. Identifica-se que, de forma geral, grande parte do modelo teórico de Simons foi validada, o que o habilita como um construto a ser estudado empiricamente em pesquisas brasileiras (Oyadomari et al., 2009).

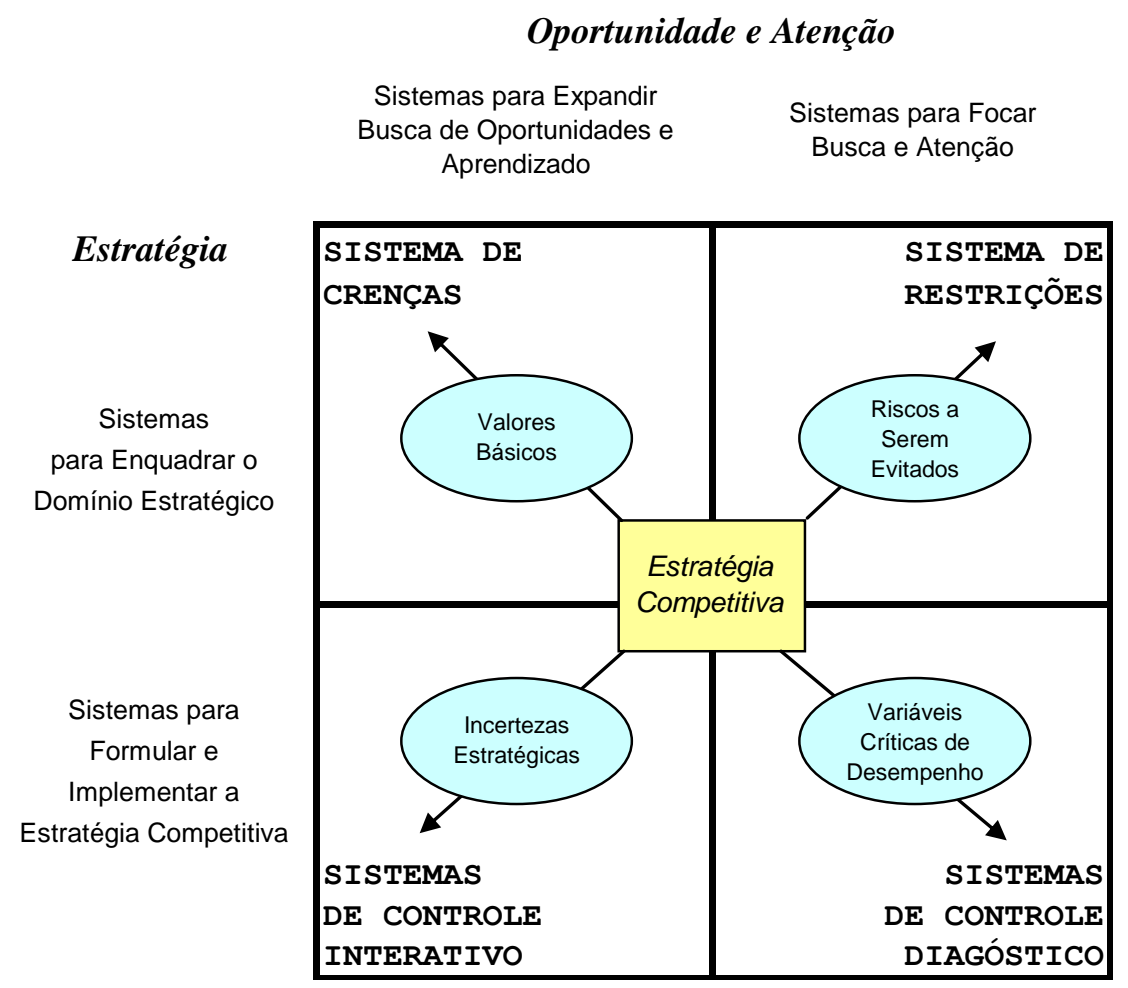

Figura 1 - Inter-relações das Alavancas de Controle com Estratégia, Oportunidade e Atenção.

Fonte: Simons (1995).

\section{INOVAÇÃO}

Inovação é geralmente definida como a adoção de uma ideia ou comportamento, relativo a um produto, serviço, dispositivo, sistema, política ou programa, que é novo para a organização 
(Damanpour \& Gopalakrishnan, 2001). Além dessa, há muitas outras definições de inovação. Para a Organisation for Economic Co-operation and Development (OECD, 2005), por exemplo, uma inovação é a implementação de um novo produto (bem ou serviço) ou processo, ou de um produto ou processo significativamente melhorado, ou de um novo método de marketing, ou de um novo método organizacional na prática de negócios, na organização do local de trabalho ou relações externas.

O trabalho de Joseph Schumpeter influenciou enormemente as teorias de inovação. Ele argumentou que o desenvolvimento econômico é impulsionado pela inovação através de um processo dinâmico no qual novas tecnologias substituem as antigas, um processo que ele chamou de destruição criativa (OECD, 2005). Schumpeter afirmou que uma mudança em ciência e tecnologia era interessante somente na sua habilidade de transformar o mundo exterior e que essa capacidade de transformação tinha que operar através da mediação do mercado. Baseado nessa premissa fundamental, ele fez a distinção entre invenção e inovação em que inovação foi uma invenção que foi adotada e comercialmente desenvolvida (Roberts, 1998). O enfoque de inovação como agente de mudança também é reforçado por Drucker (2002), que definiu inovação como o esforço para criar uma mudança objetivamente focada no potencial econômico ou social de um empreendimento.

Os processos de inovação podem ser os mais importantes da organização para a sustentação da vantagem competitiva (Kaplan \& Norton, 2004). O grande desafio para as pequenas empresas ou novos entrantes é pensar em novas ideias estratégicas. Por outro lado, o grande desafio para as empresas estabelecidas é organizacional: elas precisam desenvolver a cultura, mentalidade e ambiente subjacente para continuamente questionar o sucesso corrente e ao mesmo promover uma experimentação continuada (Markides, 1998). Sem inovação, a proposição de valor da empresa pode eventualmente ser imitada, resultando em competição apenas com base no preço (Kaplan \& Norton, 2004). Os competidores conseguem informação detalhada de $70 \%$ de todos os novos produtos um ano após o seu desenvolvimento. Na média, imitações custam um terço do que as inovações e são um terço mais rápidas (Ghemawat, 1986) Até mesmo os processos novos são rapidamente difundidos no mercado. Por isso, as estratégias devem ser tratadas como se fossem temporárias. Como explica Brown e Eisenhardt (2004), a vantagem competitiva é fugaz e, por isso, as empresas competitivas concentram-se em gerar continuamente novas fontes de vantagem. Para criar uma vantagem sustentável, você precisa ter a sorte de ter competidores que tenham um menu de opções restrito ou ser capaz de se antecipar em relação a eles (Ghemawat, 1986).

Nesse contexto de competição é que se discute a relevância do controle no processo de inovação. Embora algum grau de liberdade e flexibilidade seja essencial para equipes produtivas de inovação, a administração enfrenta o desafio de instituir mecanismos de controle que conduzam os 
projetos na direção estratégica correta e que monitorem o progresso da firma na direção das metas organizacionais e do projeto (Perez-Freije \& Enkel, 2007). Em seu estudo sobre as práticas no desenvolvimento de novos produtos, Jorgensen e Messner (2009a, p.19) mostram que o controle assume a forma de compartilhamento horizontal de informação, guiado pelos objetivos estratégicos e um entendimento geral da necessidade de ser rentável. A aplicação da prestação de contas financeiras nos pontos de controle pode ser vista como um meio de lembrar os gerentes da importância dos números contábeis - mesmo que estes sejam apenas representações imperfeitas das decisões e práticas no desenvolvimento de novos produtos (Jorgensen \& Messner, 2009a).

\section{O FRAMEWORK DE DAVILA}

As estratégias deliberadas são as estratégias pretendidas que contam com o uso diagnóstico do SCG para sua implementação. Os sistemas diagnósticos, que monitoram os resultados organizacionais, são as alavancas essenciais para a implementação das estratégias pretendidas (Simons, 1995). Conforme denominação dada por Mintzberg (1978, p. 945), as estratégias emergentes são aquelas realizadas sem que tenham sido inicialmente pretendidas; elas surgem na fase de implementação das estratégias deliberadas em virtude de decisões e oportunidades no mercado. As estratégias realizadas, portanto, contemplam as estratégias deliberadas e emergentes. As deliberadas são monitoradas pelos sistemas diagnósticos e as emergentes pelos sistemas de restrições.

As inovações podem ocorrer dentro do modelo de negócios que a empresa esteja atuando no momento, ou podem provocar uma redefinição do modelo de negócios. Inovação radical é aquela mudança significativa que afeta simultaneamente tanto o modelo de negócios quanto a tecnologia de uma empresa. Inovações radicais normalmente significam mudanças fundamentais no cenário competitivo de um setor de indústrias (Davila, Epstein, \& Shelton, 2007). Naturalmente, as inovações radicais implicam riscos altos com possibilidade de retornos maiores. As inovações incrementais, por outro lado, são uma maneira de extrair o máximo valor possível de produtos e serviços existentes sem a necessidade de fazer mudanças significativas ou grandes investimentos (Davila et al., 2007). Nesse caso, os efeitos podem ser identificados mais facilmente e envolvem menores riscos e retornos esperados também menores.

O modelo desenvolvido por Burgelman (1983) caracteriza duas ações de comportamento estratégico: as ações estratégicas induzidas e as ações estratégicas autônomas. As ações estratégicas induzidas são aquelas que são direcionadas pela alta administração da empresa consistentes com a

Revista de Administração e Inovação, São Paulo, v. 9, n. 1, p.106-125, jan./mar. 2012. 
estratégia corporativa. As ações estratégicas autônomas estão fora da estratégia corporativa em curso e podem surgir de indivíduos ou pequenos grupos sem que a alta administração tenha ciência de seu desenvolvimento. São inovações radicais que envolvem tecnologias e competências organizacionais significativamente diferentes. As inovações radicais podem também ser iniciadas pela alta administração. Markides (1998) denominou essas inovações de inovações estratégicas que representam uma nova conceituação fundamental do negócio que leva a uma forma dramaticamente diferente de jogar o jogo no negócio existente (Markides, 1998).

O framework proposto por Davila et al. (2009b) para as análises de inovação e controle sob a perspectiva da estratégia é resumida na Figura 2, que mostra os tipos de inovação e os respectivos controles. Nesse modelo, as inovações podem surgir da alta administração ou de outras partes da organização e podem ser dentro do modelo atual de negócios ou implicar um novo modelo de negócios.

O quadrante 1 está associado com o controle tradicional em que a inovação ocorre na fase de planejamento dirigido pela alta administração. Aqui as estratégias deliberadas devem ser colocadas em ação. Nesse caso, para melhorar a eficiência na execução dessas ações, a habilidade de inovação pode ficar prejudicada. De fato, no extremo, pode haver situações em que os gestores determinem o bloqueio de inovações em favor da eficiência ou segurança, evitando-se riscos. Esse é o modelo mais comum de controle gerencial no qual as ações estratégicas são implementadas e monitoradas, e desvios em relação às metas estabelecidas precisam ser corrigidas. Os sistemas diagnósticos atuam nesse quadrante.

O quadrante 2 contempla as inovações incrementais vindas da base da organização. Na medida em que as estratégias deliberadas são colocadas em ação, a dinâmica do ambiente provoca a necessidade de adaptações nas ações em curso com o aparecimento de estratégias emergentes. Há situações que exigem soluções inovadoras que os sistemas que atuam na execução da estratégia corrente não são capazes de identificar. O processo de aprendizado que capture as informações geradas na execução das estratégias emergentes através de inovações incrementais pode criar novos conhecimentos que poderão ajudar na criação de valor nos negócios da empresa. Os sistemas de controle que ajudam no aperfeiçoamento dos processos organizacionais existentes são os que oferecem a possibilidade de capturar novos conhecimentos estimulando as inovações incrementais. Entre esses sistemas, encontram-se os sistemas de qualidade, como os círculos de qualidade, e os sistemas de desenvolvimento de produtos, em que os conceitos de custo-meta ou kaizen são comumente encontrados. 


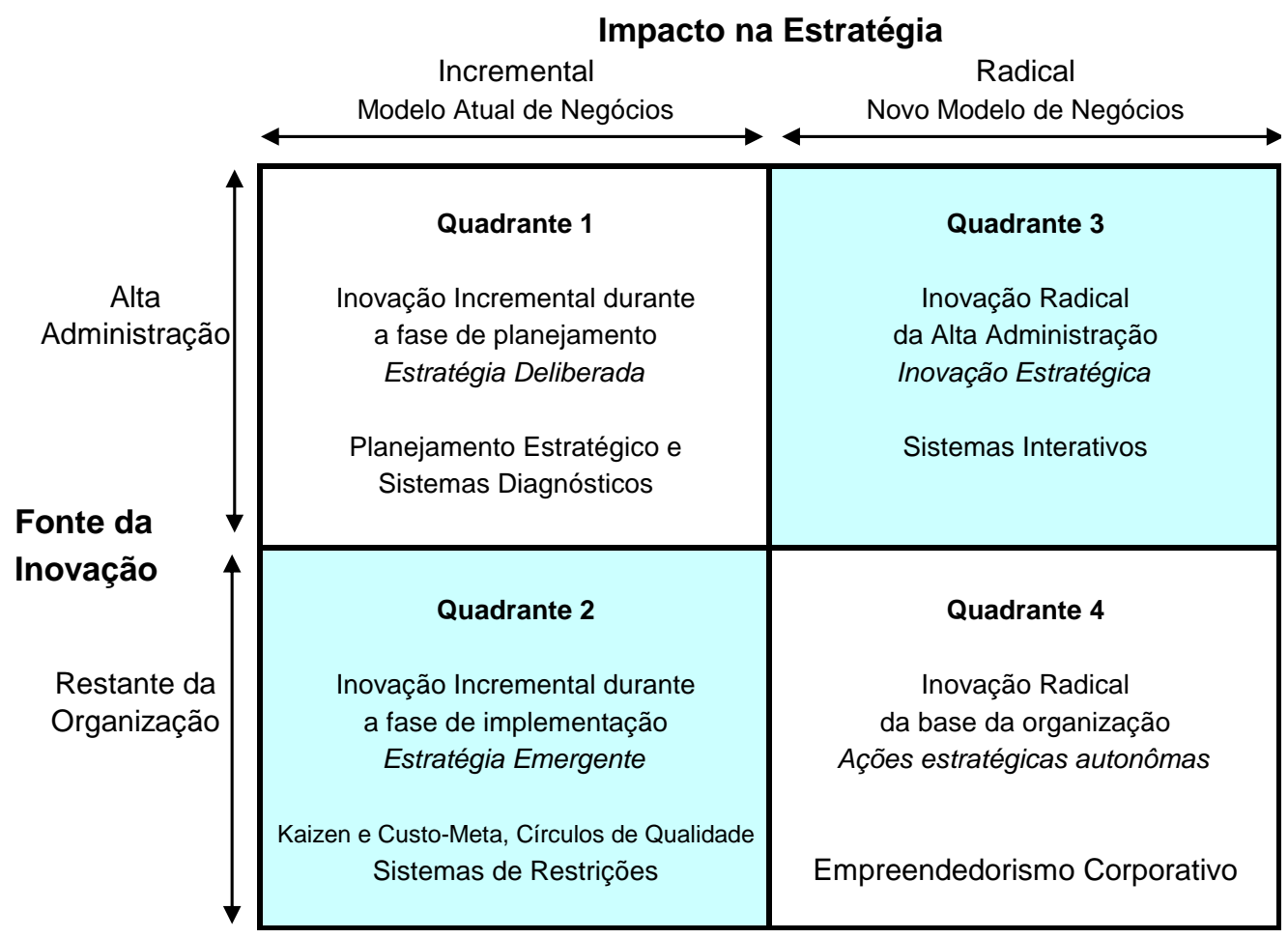

Figura 2 - Tipos de inovações e controle.

Fonte: Davila et al. (2009b).

O quadrante 3 identifica as inovações radicais vindas da alta administração, ou seja, as inovações estratégicas. Nesse contexto estratégico, além de estimular as inovações radicais no restante da organização, a alta administração deve avaliar a necessidade de mudanças radicais e as oportunidades de formular estratégias oriundas de inovações radicais. Os sistemas interativos nos quais a alta administração se envolve regularmente e pessoalmente com os seus subordinados permitem a discussão sobre as incertezas estratégicas do modelo atual de negócios. A literatura atual sobre as inovações estratégicas ainda é escassa. Do ponto de vista de estrutura organizacional, a literatura sobre estratégia apresenta algumas alternativas, como o desenvolvimento de organizações ambidestras, criação de divisões separadas ou até a terceirização desse tipo de inovação Davila et al. (2009b).

O quadrante 4 inclui as ações estratégicas autônomas que trazem as inovações radicais da base da organização. Como a possibilidade de êxito dessas inovações é menor, a empresa que desejar incentivar esse tipo de inovação precisa criar condições apropriadas que encorajem a experimentação, descoberta, exceções (Davila, 2005). Na literatura de estratégia, esse fenômeno é tratado como empreendedorismo corporativo. As pesquisas em relação aos sistemas de controle para incentivar e propiciar novas inovações radicais são muito poucas. Os sistemas de crenças, os sistemas de controle para monitorar o ambiente externo, os sistemas de avaliação de desempenho, os incentivos intrínsecos 
e extrínsecos e os sistemas de alocação de recursos podem potencialmente desempenhar um papel crítico para suportar o empreendedorismo corporativo Davila et al. (2009b).

\section{ESTUDOS PRECEDENTES}

Foram analisados diversos estudos envolvendo pesquisas que relacionam controles com processos de inovação, alguns especificamente com o processo de desenvolvimento de novos produtos. A seguir são relacionados dez estudos, resumindo-se os objetivos de pesquisa desenvolvida e os principais resultados alcançados.

a) Abernethy, M. A., \& Brownell, P. (1997). Management control systems in research and development organizations: the role of accounting, behavior and personnel controls. Accounting, Organizations and Society, 22(3/4), 233-248.

Esse estudo empírico teve como objetivo examinar o papel dos controles contábeis e não contábeis em ambientes de pesquisa e desenvolvimento. Os controles são agrupados em controles contábeis (accounting controls), controles comportamentais (behavior controls) e controles de pessoal (personnel controls). A principal contribuição dessa pesquisa foi a conclusão de que os controles não contábeis, em especial os controles de pessoal, contribuem para a efetividade organizacional, particularmente onde as características das atividades não se mostram adequadas aos controles contábeis. Os controles contábeis, por sua vez, apresentam efeitos positivos significativos nos ambientes em que a incerteza das tarefas é baixa. Os controles do tipo programáveis, tais como os controles contábeis e comportamentais, parecem inadequados onde a quantidade de exceções nos processos é elevada.

b) Davila, T. (2000). An empirical study on the drivers of management control systems' design in new product development. Accounting, Organizations and Society, 25(4/5), 383-409.

Davila investiga como as empresas adaptam os sistemas componentes do SCG para as características específicas dos processos de desenvolvimento de seus produtos. Os resultados confirmam a relevância do SCG no processo de desenvolvimento do produto e que os gerentes utilizam o SCG para obter informações necessárias para a redução de incertezas. Esses resultados diferem dos estudos anteriores que indicavam que os sistemas de controle são ferramentas para a redução de divergências de objetivos e que são apenas marginalmente relevantes para o processo de 
desenvolvimento de produtos. Informações sobre custos e projetos têm um efeito positivo sobre o desempenho. Em contrapartida, informações sobre tempo atrapalham o desempenho, justificando o argumento de que muita ênfase nos sistemas formais limita a inovação. Esse estudo fornece evidência suportando a teoria de contingência do SCG no desenvolvimento de produto. Em particular, o alinhamento entre o desenho e o uso desses sistemas e a estratégia de produto está significativamente relacionado com o desempenho.

c) Bisbe, J., \& Otley, D. (2004). The effects of the interactive use of management control systems on product innovation. Accounting, Organizations and Society, 29(8), 709-737.

O trabalho de Bisbe e Otley teve como objetivo discriminar os diferentes efeitos do uso interativo do SCG na inovação de produtos e desempenho organizacional. Os resultados não confirmaram a tese de que o uso interativo do SCG favorece a inovação de produtos. No caso de empresas com menor nível de inovação, verificou-se que o efeito do uso interativo na inovação é positivo, por outro lado, no caso de empresas com alto nível de inovação o efeito é negativo. Justificase o efeito positivo nas empresas de menor nível de inovação, pois o uso interativo do SCG pode propiciar estímulos na tomada de iniciativas de inovação e também como forma de legitimação de iniciativas autônomas. Nas empresas altamente inovadoras, a exposição e o compartilhamento das ideias através do uso interativo do SCG parecem provocar uma filtragem de iniciativas levando a uma redução da inovação. Além disso, as evidências não indicaram nenhum efeito indireto no desempenho, mas os resultados indicam que quanto mais interativamente o SCG formal é utilizado pela alta administração, maior o efeito positivo da inovação dos produtos no desempenho da organização.

d) Henri, J.-F. (2006). Management control systems and strategy: A resource-based perspective. Accounting, Organizations and Society, 31(6), 529-558.

O trabalho de Henri teve como objetivo analisar as relações entre o uso do SCG e as competências organizacionais, tendo como alicerce a teoria baseada em recursos (RBV - resourcebased view). A inovação, a aprendizagem organizacional, a orientação a mercado e o empreendedorismo são reconhecidos como competências primárias para conseguir a vantagem competitiva e promover alteração mercadológica. Os resultados sugerem que o sistema de avaliação de desempenho utilizado interativamente influencia positivamente o desenvolvimento das competências organizacionais e negativamente quando utilizado de forma diagnóstica. De modo geral, os resultados confirmam a visão de que os sistemas de controle contribuem com a implementação de estratégias planejadas e também estimulam as estratégias novas emergentes. Henri sugere que a tensão dinâmica 
pode representar uma competência e uma fonte de vantagem competitiva. Mais especificamente, essa competência pode ser representada pela habilidade de estabelecer um equilíbrio entre os dois usos, interativo e diagnóstico, do sistema de avaliação de desempenho que podem estimular a inovação ao mesmo tempo que se busca o cumprimento de metas.

e) Bisbe, J., \& Malagueño, R. (2009). The choice of interactive control systems under different innovation management modes. European Accounting Review, 18(2), 371-405.

O objetivo dessa pesquisa foi examinar se os fatores organizacionais sistemáticos levam os gerentes a fazer escolhas diferentes quanto ao SCG selecionado para o uso interativo. Além disso, os autores investigam se a adequação esperada entre o SCG selecionado para uso interativo e o modo específico de gestão de inovação é efetivamente traduzida em implicações benéficas para os resultados da inovação de produtos. As evidências obtidas indicam uma validação parcial de que a escolha de um SCG específico a ser usado interativamente está associada com o modo de gestão da inovação que fornece uma adequação suplementar. Essa adequação, entretanto, não conduz a uma habilidade melhorada para mitigar os excessos disfuncionais da força de inércia que faz com que a organização mantenha a tendência inovadora ou não inovadora. Ao contrário, a pesquisa mostra que essa adequação reforça a tendência existente, ou seja, as empresas altamente inovadoras podem ter maiores níveis de inovação e as menos inovadoras, níveis ainda menores.

f) Revellino, S., \& Mouritsen, J. (2009). The multiplicity of controls and the making of innovation. European Accounting Review, 18(2), 341-369.

A literatura sugere que os SCG influenciam a inovação, mas não especificam como ocorre essa influência. Essa pesquisa pergunta como os sistemas de controle gerencial estão envolvidos no desenvolvimento da inovação e tenta mostrar como os controles interferem e transformam a inovação. Os autores mostram que a inovação passa por diferentes processos, cada um exigindo controles diferentes que façam a mediação entre a inovação e o seu ambiente. Por isso, ao invés de assumir que todo processo pode ser estabelecido no início, ou mesmo que a inovação é um processo único, os autores propõem que a inovação seja mediada por várias tecnologias de gestão, cada uma associando a inovação com um desafio do seu ambiente. Todos os atores que participam do processo podem aprender, compartilhar, integrar e interagir, de forma que a inovação se adapte a todas as eventuais intervenções. Essas intervenções são os desafios que devem ser o foco do sistema de gestão. 
g) Chiesa, V., Frattini, F., Lamberti, L., \& Noci, G. (2009). Exploring management control in radical innovation projects. European Journal of Innovation Management, 12(4), 416-443.

Trata-se de uma pesquisa exploratória com o objetivo de investigar o controle gerencial em projetos de inovação radical. Foi utilizada a abordagem de estudo de caso múltiplo envolvendo duas empresas italianas na indústria de automação residencial. Os autores revelam a seguinte evolução do tipo de controle ao longo do processo: no estágio de criação conceitual, caracterizada por alta incerteza e menor nível de análise e separação de tarefa, há predominância de sistemas de controle suave, como os sistemas de crenças e sistemas de restrições para favorecer a criatividade e inovação; e consistentemente com a teoria de Simons, os controles interativos estão difusos para enfrentar a incerteza. No estágio de desenvolvimento, o conceito é congelado e inicia-se um procedimento mais padronizado; movendo-se lentamente para os sistemas mais diagnósticos, contudo, ainda sem deixar de usar os controles interativos. Finalmente, no estágio de comercialização, com uma margem limitada de incerteza, e com tarefas tipicamente mais analisáveis, os controles interativos diminuem em relevância e os controles diagnósticos tornam-se preeminentes.

h) Davila, A., Foster, G., \& Li, M. (2009). Reasons for management control systems adoption: Insights from product development choice by early-stage entrepreneurial companies. Accounting, Organizations and Society, 34(3/4), 322-347.

Essa pesquisa examina a adoção de SGC no processo de desenvolvimento de produto. Os SCG considerados nesse estudo são os sistemas formais específicos para o desenvolvimento de produtos, incluindo: gerência de projetos por pontos de controle (project milestones), orçamento para projetos de desenvolvimento, relatórios comparativos do progresso atual contra o planejado, processo de seleção de projetos, roteiro da carteira de produtos, processo de testes dos conceitos dos produtos, e as diretrizes da composição de equipes de projetos. A pesquisa identificou seis razões para a adoção de um SCG. Duas razões por motivos externos: legitimação da empresa e contratos com parceiros externos. Das quatro razões internas, duas são proativas: formação dos gerentes e foco na execução da estratégia, e duas são reativas: reação a problemas e codificação do aprendizado (associado com a formalização dos processos repetitivos). As evidências apresentadas pelos autores fornecem dados qualitativos consistentes com o papel dos SCG que incluem o estímulo ao diálogo e criação de ideias; controle da execução através dos sistemas diagnósticos; e estabilização do ambiente que, pela natureza do processo de inovação, é cheia de oportunidades. 
i) Jorgensen, B., \& Messner, M. (2009b). Management control in new product development: the dynamic of managing flexibility and efficiency. Journal of Management Accounting Research, 21(1), 99-124.

Essa pesquisa mostra como diversas regras e procedimentos formais são utilizados para controlar o processo de desenvolvimento de produto e discute os tipos de formalizações que são caracterizados como controles habilitadores. Esse conceito foi introduzido por Adler e Borys (1996) em oposição aos controles coercivos. O modelo de estágios de controle (stage-gate model) é particularmente importante, pois permite a troca formal de atividades rotineiras por atividades não rotineiras ou até a separação de atividades. Os autores concluem que o comprometimento com o processo de controle habilitador e não coercivo permitiu à organização equilibrar a eficiência e flexibilidade no processo de desenvolvimento de produtos de forma que nem a alta administração nem os engenheiros e gerentes sintam falta de atenção. A evidência sugere que a arquitetura de controle analisada constitui uma configuração harmoniosa já que não havia insatisfação na combinação existente entre eficiência e flexibilidade.

j) Akroyd, C., Narayan, S., \& Sridharan, V. G. (2009). The use of control systems in new product development innovation: advancing the "help or hinder" debate. The IUP Journal of Knowledge Management, 7(5/6), 70-90.

Os autores sugerem que cada tipo de projeto de inovação de desenvolvimento de novos produtos pode requerer o uso de um processo de stage-gate (estágios com pontos de controle). Os autores concluem que o modelo departamental, no qual as atividades são executadas sequencialmente dentro de cada departamento durante cada estágio do processo, pode ser mais adequado para os projetos semirradicais com alto nível de incerteza tecnológica. O modelo atividade/decisão, que é formado por conjuntos de atividades que são agrupados em estágios seguidos de decisão gerencial nos pontos de controle, é mais adequado para os projetos semirradicais com alto nível de incerteza mercadológica e para projetos incrementais. E o modelo conversão/resposta que também considera atividades, embora elas não sejam designadas a estágios ou pontos de controle específicos e sejam executadas quando necessárias, é mais adequado para os projetos radicais.

\section{SÍNTESE DOS ESTUDOS ANALISADOS}

O Quadro 1 apresenta uma síntese dos estudos analisados indicando a forma de pesquisa e os principais resultados obtidos.

Revista de Administração e Inovação, São Paulo, v. 9, n. 1, p.106-125, jan./mar. 2012. 


\begin{tabular}{|c|c|c|}
\hline Pesquisa & Forma de Pesquisa & Principais resultados \\
\hline $\begin{array}{l}\text { Abernethy \& } \\
\text { Brownell, } 1997\end{array}$ & $\begin{array}{l}\text { Questionário e } \\
\text { entrevistas : } 127 \\
\text { respondentes }\end{array}$ & $\begin{array}{l}\text { Os controles de pessoal contribuem para a efetividade } \\
\text { organizacional. Os controles contábeis apresentam efeitos } \\
\text { positivos significativos nos ambientes em que a incerteza das } \\
\text { tarefas é baixa. }\end{array}$ \\
\hline Davila, 2000 & $\begin{array}{l}\text { Estudos de casos em } \\
12 \text { unidades de } \\
\text { negócios de } 7 \text { cias. e } \\
\text { levantamento com } \\
56 \text { respondentes }\end{array}$ & $\begin{array}{l}\text { Confirma a relevância do SCG no processo de desenvolvimento } \\
\text { do produto; os gerentes utilizam o SCG para obter informações } \\
\text { para a redução de incertezas. Informações sobre custos e projetos } \\
\text { têm um efeito positivo sobre o desempenho. Muita ênfase nos } \\
\text { sistemas formais limita a inovação. }\end{array}$ \\
\hline $\begin{array}{l}\text { Bisbe \&e } \\
\text { Otley, } 2004\end{array}$ & $\begin{array}{l}\text { Levantamento por } \\
\text { questionário: } 58 \\
\text { respondentes }\end{array}$ & $\begin{array}{l}\text { Os resultados não confirmaram a tese de que o uso interativo do } \\
\text { SCG favorece a inovação de produtos. No caso de empresas com } \\
\text { menor nível de inovação, verificou-se que o efeito do uso } \\
\text { interativo na inovação é positivo, por outro lado, no caso de } \\
\text { empresas com alto nível de inovação o efeito é negativo. }\end{array}$ \\
\hline Henri, 2006 & $\begin{array}{l}\text { Questionários: } 383 \\
\text { respondentes numa } \\
\text { amostra de } 2175\end{array}$ & $\begin{array}{l}\text { O sistema de avaliação de desempenho utilizado interativamente } \\
\text { influencia positivamente as competências organizacionais e } \\
\text { negativamente quando utilizado de forma diagnóstica. A } \\
\text { habilidade de estabelecer um equilíbrio entre os dois usos, } \\
\text { interativo e diagnóstico, pode estimular a inovação. }\end{array}$ \\
\hline $\begin{array}{l}\text { Bisbe \& } \\
\text { Malagueño, } \\
2009\end{array}$ & $\begin{array}{l}\text { Questionário com } \\
57 \text { empresas }\end{array}$ & $\begin{array}{l}\text { A similaridade nos padrões entre o modo de gestão da inovação e } \\
\text { os sistemas de controle não conduzem a um impacto benéfico no } \\
\text { nível de produção de inovação. O uso de sistemas de controle } \\
\text { interativo pode fazer com que as empresas altamente inovadoras } \\
\text { possam ter maiores níveis de inovação e as menos inovadoras, } \\
\text { níveis ainda menores. }\end{array}$ \\
\hline $\begin{array}{l}\text { Revellino \& } \\
\text { Mouritsen, } \\
2009\end{array}$ & $\begin{array}{l}\text { Estudo de caso com } \\
\text { base na inovação } \\
\text { Telepass (sistema de } \\
\text { pedágio) }\end{array}$ & $\begin{array}{l}\text { A inovação passa por diferentes processos, cada um exigindo } \\
\text { controles diferentes que façam a mediação entre a inovação e o } \\
\text { seu ambiente. Os autores propõem que a inovação seja mediada } \\
\text { por várias tecnologias de gestão, cada uma associando a inovação } \\
\text { com um desafio do seu ambiente. }\end{array}$ \\
\hline $\begin{array}{l}\text { Chiesa et al., } \\
2009\end{array}$ & $\begin{array}{l}\text { Estudo de caso } \\
\text { múltiplo envolvendo } \\
\text { duas empresas } \\
\text { italianas com dois } \\
\text { projetos de inovação } \\
\text { de cada empresa }\end{array}$ & $\begin{array}{l}\text { No estágio de criação conceitual, há predominância dos sistemas } \\
\text { de crenças e sistemas de restrições. No estágio de } \\
\text { desenvolvimento, um procedimento mais padronizado; movendo- } \\
\text { se para os sistemas mais diagnósticos. No estágio de } \\
\text { comercialização, os controles interativos diminuem em relevância } \\
\text { e os controles diagnósticos tornam-se preeminentes. }\end{array}$ \\
\hline $\begin{array}{l}\text { Davila, Foster, } \\
\text { Li (2009a) }\end{array}$ & $\begin{array}{l}\text { Entrevistas e } \\
\text { questionários }\end{array}$ & $\begin{array}{l}\text { A pesquisa identificou seis razões para adoção de um SCG: } \\
\text { legitimação da companhia, contratos com parceiros externos, } \\
\text { formação dos gerentes, necessidade de foco na execução da } \\
\text { estratégia, reação a problemas e codificação do aprendizado. O } \\
\text { gerenciamento de projetos através de pontos de controle (project } \\
\text { milestones) é o sistema que a maioria das empresas adota. }\end{array}$ \\
\hline $\begin{array}{l}\text { Jorgensen \& } \\
\text { Messner, } \\
\text { 2009b }\end{array}$ & $\begin{array}{l}\text { Estudo de caso com } \\
\text { base em entrevistas, } \\
\text { dados históricos e } \\
\text { observação }\end{array}$ & $\begin{array}{l}\text { O modelo de estágios de controle (stage-gate model) permite a } \\
\text { troca formal de atividades rotineiras por atividades não rotineiras. } \\
\text { O comprometimento com o processo de controle habilitador e não } \\
\text { coercivo permitiu à organização equilibrar a eficiência e } \\
\text { flexibilidade no processo de desenvolvimento de produtos. }\end{array}$ \\
\hline
\end{tabular}

Revista de Administração e Inovação, São Paulo, v. 9, n. 1, p.106-125, jan./mar. 2012. 


\begin{tabular}{l|l|l}
\hline & $\begin{array}{l}\text { A pesquisa considera o processo de stage-gate como SCG para a } \\
\text { gestão do fluxo de novos produtos. O modelo departamental é }\end{array}$ \\
$\begin{array}{l}\text { Akroyd et al., } \\
\text { mais apropriado para os projetos de inovação semirradicais com }\end{array}$ & $\begin{array}{l}\text { Estudo de Caso } \\
\text { através de } \\
\text { observação } \\
\text { participante }\end{array}$ & $\begin{array}{l}\text { alto nível de incerteza tecnológica. O modelo atividade/decisão } \\
\text { para projetos semirradicais com alto nível de incerteza } \\
\text { mercadológica e modelo conversão/resposta para projetos } \\
\text { radicais. }\end{array}$ \\
\hline
\end{tabular}

Quadro 1 - Síntese dos Estudos Analisados.

Fonte: Elaborado pelos autores.

\section{CONSIDERAÇÕES FINAIS}

Como pode ser observado nos diversos estudos analisados, o modelo de controle de Simons tem sido utilizado nas pesquisas envolvendo controles gerenciais e processo de inovação. O conceito do uso interativo tem sido explorado em diversos estudos (Bisbe \& Malagueño, 2009; Bisbe; \& Otley, 2004; Henri, 2006). As diferentes alavancas definidas por Simons (1995) podem estar associadas com diferentes processos ou fases da inovação (Chiesa et al., 2009; Revellino \& Mouritsen, 2009) e diferentes controles podem ser mais adequados conforme o tipo de projeto de inovação (Akroyd et al., 2009). Alguns SCG específicos podem ser mais relevantes no processo de inovação (Jorgensen \& Messner, 2009b). As evidências empíricas das pesquisas internacionais apresentam resultados difusos, mas podem permitir novas pesquisas visando ampliar o entendimento em relação ao processo de inovação e controle. Davila (2000) confirmou a relevância do SCG no processo de desenvolvimento de produto. Bisbe e Otley (2004) verificaram que o efeito do uso interativo na inovação é positivo em empresas com menor nível de inovação, embora não tenham confirmado a tese de que o uso interativo do SCG favorece a inovação de produtos. Henri (2006) mostra que o equilíbrio entre os usos interativo e diagnóstico pode estimular a inovação. Bisbe e Malagueño (2009) apresentam evidências que suportam parcialmente as hipóteses de associação entre o modo de gestão da inovação com o uso interativo de SCG. Chiesa et al. (2009) mostra a associação de diferentes abordagens do SCG com as diferentes fases de um projeto de inovação. Revellino e Mouritsen (2009) mostram que a inovação passa por diferentes processos, cada um exigindo controles diferentes que façam a mediação entre a inovação e o seu ambiente.

Nesse contexto, o framework de Davila et al. (2009b) torna-se particularmente relevante para explorar os efeitos dos instrumentos de controle em ambientes de inovação. Essa estrutura conceitual, baseada na ideia de que a inovação é um processo multifacetado, identifica quatro tipos potenciais de sistemas de controle que são distintos nas várias dimensões, tais como seus propósitos, fontes de informação, necessidades de coordenação, ou incentivos sociais e econômicos (Davila et al., 2009b). Revista de Administração e Inovação, São Paulo, v. 9, n. 1, p.106-125, jan./mar. 2012. 
Sugere-se assim que futuras pesquisas sejam realizadas, notadamente empíricas, que caracterizem os relacionamentos entre os diferentes SCG e os diferentes processos de inovação. Essas pesquisas poderão contribuir para avançar na compreensão das estruturas e usos de SCG com o objetivo de estimular o processo de inovação e que consequentemente apresente influência positiva no desempenho organizacional.

\section{REFERÊNCIAS}

Abernethy, M. A., \& Brownell, P. (1997). Management control systems in research and development organizations: the role of accounting, behavior and personnel controls. Accounting, Organizations and Society, 22(3/4), 233-248.

Adler, P., \& Borys, B. (1996). The two types of bureaucracy: enabling and coercive. Administrative Science Quarterly, 41(1), 61-89.

Akroyd, C., Narayan, S., \& Sridharan, V. G. (2009). The use of control systems in new product development innovation: advancing the "help or hinder" debate. The IUP Journal of Knowledge Management, 7(5/6), 70-90.

Anthony, R. N., \& Govidarajan, V. (2008). Sistemas de controle gerencial (12a. ed.). São Paulo: McGraw-Hill.

Bisbe, J., \& Malagueño, R. (2009). The choice of interactive control systems under different innovation management modes. European Accounting Review, 18(2), 371-405.

Bisbe, J., \& Otley, D. (2004). The effects of the interactive use of management control systems on product innovation. Accounting, Organizations and Society, 29(8), 709-737.

Brown, S. L., \& Eisenhardt, K. M. (2004). Estratégia competitiva no limiar do caos: uma visão dinâmica para as transformações corporativas. São Paulo: Cultrix.

Burgelman, R. A. (1983). A model of the interaction of strategic behavior, corporate context, and the concept of strategy. Academy of Management Review, 8(1), 61-70.

Chiesa, V., Frattini, F., Lamberti, L., \& Noci, G. (2009). Exploring management control in radical innovation projects. European Journal of Innovation Management, 12(4), 416-443.

Damanpour, F., \& Gopalakrishnan, S. (2001). The dynamics of the adoption of product and process innovations in organizations. Journal of Management Studies, 38(1), 45-65.

Davila, A., Foster, G., \& Li, M. (2009a). Reasons for management control systems adoption: Insights from product development choice by early-stage entrepreneurial companies. Accounting, Organizations and Society, 34(3/4), 322-347. 
Davila, A., Foster, G., \& Oyon, D. (2009b). Accounting and control, entrepreneurship and innovation: venturing into new research opportunities. European Accounting Review, 18(2), 281-311.

Davila, A., \& Oyon, D. (2009). Introduction to the special section on accounting, innovation and entrepreneurship. European Accounting Review, 18(2), 277-280.

Davila, T. (2005). The promise of management control systems for innovation and strategic change. In C. S. Chapman (Ed.), Controlling strategy: management, accounting, and performance measurement (pp. 37-61). Oxford: Oxford University Press

Davila, T. (2000). An empirical study on the drivers of management control systems' design in new product development. Accounting, Organizations and Society, 25(4/5), 383-409.

Davila, T., Epstein, M. J., \& Shelton, R. (2007). As regras da inovação: como gerenciar, como medir e como lucrar. Porto Alegre: Bookman.

Drucker, P. F. (2002). The discipline of innovation. Harvard Business Review, 80(8), 95-104.

Frezatti, F., Rocha, W., Nascimento, A. R., \& Junqueira, E. (2009). Controle gerencial: uma abordagem da contabilidade gerencial no contexto exonômico, comportamental e sociológico. São Paulo: Atlas.

Ghemawat, P. (1986). Sustainable advantage. Harvard Business Review, 64(5), 53-59.

Hall, M. (2010). Accounting information and managerial work. Accounting, Organizations and Society, 35(3), 301-315.

Henri, J.-F. (2006). Management control systems and strategy: a resource-based perspective. Accounting, Organizations and Society, 31(6), 529-558.

Jorgensen, B., \& Messner, M. (2009a). Accounting and strategising: a case study from new product development. Accounting, Organizations and Society, 35(2), 184-204.

Jorgensen, B., \& Messner, M. (2009b). Management control in new product development: the dynamic of managing flexibility and efficiency. Journal of Management Accounting Research, 21(1), 99124.

Kaplan, R. S., \& Norton, D. P. (2004). Mapas estratégicos - balanced scorecard: convertendo ativos intangíveis em resultados tangíveis. Rio de Janeiro: Elsevier.

Markides, C. (1998). Strategic innovation in established companies. Sloan Management Review, 39(3), $31-42$.

Merchant, K. A. \& Van Der Stede, W. A. (2007) Management control systems: performance measurement, evaluation and incentives ( $2^{\text {nd }}$ ed.). Harlow: FT Prentice-Hall.

Mintzberg, H. (1978). Patterns in strategy formation. Management Science, 24(9), 934-948. 
Organisation for Economic Co-operation and Development. (2005). Oslo manual: guidelines for collecting and interpreting innovation data ( $3^{\text {rd }}$ ed.). Paris: Author.

Otley, D. (2003). Management control and performance management: whence and whither? The British Accounting Review, 35(4), 309-326.

Oyadomari, J. C T., Frezatti, F., Cardoso, R. L., \& Aguiar, A. B. (2009). Análise dos trabalhos que usaram o modelo levers of control de Simons na literatura internacional no período de 1995 a 2007. Revista de Contabilidade e Organizações, 3(7), 25-42.

Perez-Freije, J., \& Enkel, E. (2007). Creative tension in the innovation process: how to support the right capabilities. European Management Journal, 25(1), 11-24.

Porter, M. E. (1989). Vantagem competitiva: criando e sustentando um desempenho superior. Rio de Janeiro: Elsevier.

Revellino, S., \& Mouritsen, J. (2009). The multiplicity of controls and the making of innovation. European Accounting Review, 18(2), 341-369.

Roberts, R. (1998). Managing innovation: the pursuit of competitive advantage and the design of innovation intense environments. Research Policy, 27(2), 159-175.

Simons, R. (2000). Performance measurement \& control systems for implementing strategy. Upper Saddle River: Prentice-Hall.

Simons, R. (1995). Levers of control: how managers use innovative control systems to drive strategic renewal. Boston: Harvard Business School Press. 


\title{
MANAGEMENT CONTROL SYSTEMS AND THE INNOVATION PROCESS
}

\begin{abstract}
This research aims to identify the contributions of the international empirical studies for the development of Brazilian researches in the analyses of the relationships between the Management Control Systems (MCS) and the innovation. The innovation is a process and as such has to be managed. This need for the innovation management emphasizes the relevance of the control systems because the suitability of the managerial controls in the different strategic contexts may be fundamental for the success of the organizations. This paper makes a brief review of the Simons (1995)'s Levers of Control model which is one of the most comprehensive organizational management models. Pointing out the importance of innovation in the organizational management, the paper shows a brief definition of innovation and makes a review of the recently published literature in the main international journals involving MCS and the innovation process. It is noticed that the theme is absent in the local literature, and internationally it is noticed a growing number of researches, however, the results are still diffused, which offer the possibility of exploring new opportunities of researches. In this context, the framework presented by Davila, Foster \& Oyon (2009b) for analyses of controls and innovations under the strategic perspective provides a framework to advance in the researches aiming to analyze different ways of managerial controls that can stimulate the innovation and consequent positive effect on the organizational performance.
\end{abstract}

Key-words: Innovation; Management Control Systems; Levers of Control.

Data do recebimento do artigo: 09/04/2011

Data do aceite de publicação: 05/08/2011

Revista de Administração e Inovação, São Paulo, v. 9, n. 1, p.106-125, jan./mar. 2012. 